\title{
Entropy, stochastic matrices, and quantum operations
}

\author{
Lin Zhang* \\ Institute of Mathematics, Hangzhou Dianzi University, Hangzhou 310018, PR China
}

\begin{abstract}
The goal of the present paper is to derive some conditions on saturation of (strong) subadditivity inequality for the stochastic matrices. The notion of relative entropy of stochastic matrices is introduced by mimicking quantum relative entropy. Some properties of this concept are listed and the connection between the entropy of the stochastic quantum operations and that of stochastic matrices are discussed.
\end{abstract}

Keywords: Quantum operation; entropy; stochastic matrix

\section{Introduction}

If the column vectors

$$
\mathbf{p}=\left[p_{1}, \ldots, p_{N}\right]^{\top} \in \mathbb{R}^{N}, \quad \mathbf{q}=\left[q_{1}, \ldots, q_{N}\right]^{\top} \in \mathbb{R}^{N}
$$

are two probability distributions, the Shannon entropy of $\mathbf{p}$ is defined by

$$
\mathrm{H}(\mathbf{p}) \stackrel{\text { def }}{=}-\sum_{i=1}^{N} p_{i} \log _{2} p_{i},
$$

where $x \log _{2} x$ is set to 0 if $x=0$, and the relative entropy of $\mathbf{p}$ and $\mathbf{q}$ is defined by

$$
\mathrm{H}(\mathbf{p} \| \mathbf{q}) \stackrel{\text { def }}{=} \sum_{i=1}^{N} p_{i} \log _{2} \frac{p_{i}}{q_{i}}
$$

when $\mathbf{p}$ is absolute continuously with respect to $\mathbf{q} ; \mathrm{H}(\mathbf{p} \| \mathbf{q})=+\infty$ otherwise.

Let $B=\left[b_{i j}\right]$ be a $N \times N$ bi-stochastic matrix, that is, $b_{i j} \geqslant 0$, and

$$
\sum_{i=1}^{N} b_{i j}=\sum_{j=1}^{N} b_{i j}=1
$$

\footnotetext{
*E-mail: godyalin@163.com; linyz@hdu.edu.cn
} 
for each $i, j=1, \ldots, N$. Let $\pi$ be a permutation of the set $\{1, \ldots, N\}$. For any $i, j \in\{1, \ldots, N\}$, we define $c_{i j}=1$ when $i=\pi(j)$ and $c_{i j}=0$ when $i \neq \pi(j)$. Then the matrix $C=\left[c_{i j}\right]$ is called a permutation matrix. Let $\mathbf{S}_{N}$ be the set of all $N \times N$ permutation matrices and $\mathbf{B}_{N}$ be the convex hull $\mathbf{B}_{N}$ of $\mathbf{S}_{N}$. The well-known Birkhoff-von Neumann theorem indicates that $\mathbf{B}_{N}$ is the set of all $N \times N$ bi-stochastic matrix.

We only consider finite dimensional complex Hilbert spaces. A state $\rho$ of a quantum system, described by Hilbert space $\mathcal{H}$, is a positive semi-definite matrix of trace one and call it the density matrix. The set of all density matrices of $\mathcal{H}$ is denoted by $\mathrm{D}(\mathcal{H})$, if $\rho \in \mathrm{D}(\mathcal{H})$ is invertible, then $\rho$ is said to be faithful. If $\rho$ and $\sigma$ are two quantum states, then the von Neumann entropy of $\rho$ is defined by

$$
S(\rho) \stackrel{\text { def }}{=}-\operatorname{Tr}\left(\rho \log _{2} \rho\right),
$$

the quantum relative entropy between $\rho$ and $\sigma$ is defined by

$$
\mathrm{S}(\rho \| \sigma) \stackrel{\text { def }}{=} \operatorname{Tr}\left(\rho\left(\log _{2} \rho-\log _{2} \sigma\right)\right)
$$

if $\operatorname{supp}(\rho) \subseteq \operatorname{supp}(\sigma) ; \mathrm{S}(\rho \| \sigma)=+\infty$ otherwise, see [7].

Let $\mathcal{H}$ and $\mathcal{K}$ be two Hilbert spaces, $\mathrm{L}(\mathcal{H}, \mathcal{K})$ be the set of all linear operators from $\mathcal{H}$ to $\mathcal{K}$, denote $\mathrm{L}(\mathcal{H}, \mathcal{H})$ by $\mathrm{L}(\mathcal{H})$. Let $\mathrm{T}(\mathcal{H}, \mathcal{K})$ denote the set of all linear super-operators from $\mathrm{L}(\mathcal{H})$ to $\mathrm{L}(\mathcal{K})$, similarly, denote $\mathrm{T}(\mathcal{H}, \mathcal{H})$ by $\mathrm{T}(\mathcal{H})$. We say that $\Phi \in \mathrm{T}(\mathcal{H}, \mathcal{K})$ is completely positive $(\mathrm{CP})$ if for each $k \in \mathbb{N}$,

$$
\Phi \otimes \mathbb{1}_{\mathrm{M}_{k}(\mathbb{C})}: \mathrm{L}(\mathcal{H}) \otimes \mathrm{M}_{k}(\mathbb{C}) \rightarrow \mathrm{L}(\mathcal{K}) \otimes \mathrm{M}_{k}(\mathbb{C})
$$

is positive, where $\mathrm{M}_{k}(\mathbb{C})$ is the set of all $k \times k$ complex matrices. It follows from the famous theorems of Choi [2] and Kraus [3] that $\Phi$ can be represented in the form

$$
\Phi=\sum_{j} \mathbf{A d}_{M_{j}}
$$

where $\left\{M_{j}\right\}_{j=1}^{n} \subseteq \mathrm{L}(\mathcal{H}, \mathcal{K})$, that is,

$$
\Phi(X)=\sum_{j=1}^{n} M_{j} X M_{j}^{\dagger}, X \in \mathrm{L}(\mathcal{H}) .
$$

Throughout the present paper, t means adjoint operation of some operator. Denote by $\mathrm{CP}(\mathcal{H}, \mathcal{K})(\mathrm{CP}(\mathcal{H}))$ the set of all linear $\mathrm{CP}$ super-operators in $\mathrm{T}(\mathcal{H}, \mathcal{K})(\mathrm{T}(\mathcal{H}))$.

The so-called quantum operation is just a trace non-increasing $\Phi \in \mathrm{CP}(\mathcal{H}, \mathcal{K})$, if $\Phi$ is tracepreserving, then it is called stochastic; if $\Phi$ is stochastic and unit-preserving, then it is called bi-stochastic. 
The famous Jamiołkowski isomorphism

$$
J: \mathrm{T}(\mathcal{H}) \longrightarrow \mathrm{L}(\mathcal{H} \otimes \mathcal{H})
$$

transforms each $\Phi \in \mathrm{T}(\mathcal{H})$ into an operator $J(\Phi) \in \mathrm{L}(\mathcal{H} \otimes \mathcal{H})$, where

$$
J(\Phi)=\Phi \otimes \mathbb{1}_{\mathrm{L}(\mathcal{H})}\left(\operatorname{vec}\left(\mathbb{1}_{\mathcal{H}}\right) \operatorname{vec}\left(\mathbb{1}_{\mathcal{H}}\right)^{\dagger}\right) .
$$

If $\Phi \in \mathrm{CP}(\mathcal{H})$, then $J(\Phi)$ is a positive semi-definite operator, in particular, if $\Phi$ is stochastic, then $\frac{1}{N} J(\Phi)$ is a state on $\mathcal{H} \otimes \mathcal{H}$. If $\Phi \in \mathrm{CP}(\mathcal{H})$ is a stochastic quantum operation, we denote the von Neumann entropy $S\left(\frac{1}{N} J(\Phi)\right)$ of $\frac{1}{N} J(\Phi)$ by $S^{m a p}(\Phi)$ and call it the map entropy [10], which describes the decoherence induced by the quantum operation $\Phi$.

\section{On saturation of classical relative entropy}

In order to obtain the condition for saturation of classical relative entropy, we need the following lemmas.

Lemma 2.1. ([5]) Let $\mathcal{H}$ be a Hilbert space, $\rho$ and $\sigma$ be two states of $\mathcal{H}$. If $\Phi \in \mathrm{CP}(\mathcal{H})$ is stochastic, then $\mathrm{S}(\Phi(\rho) \| \Phi(\sigma)) \leqslant \mathrm{S}(\rho \| \sigma)$.

Lemma 2.2. ([4]) Let $\left\{A_{1}, \ldots, A_{k}\right\} \subseteq \mathrm{L}\left(\mathbb{C}^{n}\right)$ and $\left\{B_{1}, \ldots, B_{k}\right\} \subseteq \mathrm{L}\left(\mathbb{C}^{m}\right)$ be two commuting families of Hermitian matrices. Then there exist unitary matrices $U \in \mathrm{L}\left(\mathbb{C}^{n}\right)$ and $V \in \mathrm{L}\left(\mathbb{C}^{m}\right)$ such that $U^{\dagger} A_{j} U$ and $V B_{j} V^{\dagger}$ are diagonal matrices with diagonals $\mathbf{a}_{j}=\left[a_{1 j}, \ldots, a_{n j}\right]^{\top}$ and $\mathbf{b}_{j}=\left[b_{1 j}, \ldots, b_{m j}\right]^{\top}$, respectively, for $j=1, \ldots, k$. Then the following conditions are equivalent:

(i) There is a super-operator $\Phi \in \mathrm{CP}\left(\mathbb{C}^{n}, \mathbb{C}^{m}\right)$ such that $\Phi\left(A_{j}\right)=B_{j}(j=1, \ldots, k)$.

(ii) There is an $m \times n$ non-negative matrix $D=\left[d_{\mu \nu}\right]$ such that $\left[b_{i j}\right]=D\left[a_{i j}\right]$.

Moreover, if the statement (ii) is satisfied, then $\Phi$ is bi-stochastic if and only if $D$ is bi-stochastic.

Theorem 2.3. Let $T$ be a $N \times N$ stochastic matrix, $\mathbf{p}=\left[p_{1}, p_{2}, \ldots, p_{N}\right]^{\top}$ and $\mathbf{q}=\left[q_{1}, q_{2}, \ldots, q_{N}\right]^{\top}$ be two N-dimensional probability distributions. Then $\mathrm{H}(T \mathbf{p} \| T \mathbf{q}) \leqslant \mathrm{H}(\mathbf{p} \| \mathbf{q})$. Moreover, for each $1 \leqslant k \leqslant$ $N, p_{k}, q_{k}>0$, then $\mathrm{H}(T \mathbf{p} \| T \mathbf{q})=\mathrm{H}(\mathbf{p} \| \mathbf{q})$ if and only if the following conditions hold:

(i) $\mathbf{p}=\bigoplus_{k=1}^{K} \mu_{k} \mathbf{p}_{k} \otimes \mathbf{r}_{k}$ and $\mathbf{q}=\bigoplus_{k=1}^{K} v_{k} \mathbf{q}_{k} \otimes \mathbf{r}_{k}$, where $\mathbf{p}_{k}, \mathbf{q}_{k}$ denote the $m_{k}$-dimensional probability vectors, and $\mathbf{r}_{k}$ denotes the $n_{k}$-dimensional probability vectors, and $\mu_{k}, v_{k} \geqslant 0, k=1, \ldots, K ; \sum_{k=1}^{K} \mu_{k}=$ $\sum_{k=1}^{K} v_{k}=1, \sum_{k=1}^{K} m_{k} n_{k}=N$;

(ii) $T=\bigoplus_{k=1}^{K} \pi_{k} \otimes T_{k}, \pi_{k} \in \mathbf{S}_{m_{k}}$ and $T_{k}$ is $n_{k} \times n_{k}$ stochastic matrix for each $k=1, \ldots, K$. 
Proof. Let $\rho, \sigma, \rho^{\prime}$ and $\sigma^{\prime}$ be diagonal matrices with diagonal $\mathbf{p}, \mathbf{q}, T \mathbf{p}$ and $T \mathbf{q}$, respectively. Then it follows from the Lemma 2.2 that there is a stochastic $\Phi \in \operatorname{CP}\left(\mathbb{C}^{n}, \mathbb{C}^{m}\right)$ such that $\Phi(\rho)=\rho^{\prime}$, $\Phi(\sigma)=\sigma^{\prime}$. Note that $\mathrm{H}(\mathbf{p} \| \mathbf{q})=\mathrm{S}(\rho \| \sigma)$ and $\mathrm{H}(T \mathbf{p} \| T \mathbf{q})=\mathrm{S}\left(\rho^{\prime} \| \sigma^{\prime}\right)$, so by Lemma 1 we have $\mathrm{H}(T \mathbf{p} \| T \mathbf{q}) \leqslant \mathrm{H}(\mathbf{p} \| \mathbf{q})$. Moreover, if for each $1 \leqslant k \leqslant N, p_{k}, q_{k}>0$, then the states $\rho, \sigma, \Phi(\rho)=\rho^{\prime}$ and $\Phi(\sigma)=\sigma^{\prime}$ are faithful. If $\mathrm{H}(T \mathbf{p} \| T \mathbf{q})=\mathrm{H}(\mathbf{p} \| \mathbf{q})$, then $\mathrm{S}(\rho \| \sigma)=\mathrm{S}(\Phi(\rho) \| \Phi(\sigma))$. By a result in 6]

$$
\mathrm{S}(\Phi(\rho) \| \Phi(\sigma))=\mathrm{S}(\rho \| \sigma)
$$

if and only if the following statements hold:

(1) $\mathcal{H}$ and $\mathcal{K}$ can be decomposed by the form $\mathcal{H}=\bigoplus_{k=1}^{K} \mathcal{H}_{k}^{L} \otimes \mathcal{H}_{k}^{R}, \mathcal{K}=\bigoplus_{k=1}^{K} \mathcal{K}_{k}^{L} \otimes \mathcal{K}_{k}^{R}$, where $\operatorname{dim} \mathcal{H}_{k}^{L}=\operatorname{dim} \mathcal{K}_{k}^{L}$.

(2) If $\Phi_{k}$ is the restriction of $\Phi$ to $\mathrm{L}\left(\mathcal{H}_{k}^{L} \otimes \mathcal{H}_{k}^{R}\right)$, then $\Phi_{k} \in \mathrm{T}\left(\mathcal{H}_{k}^{L} \otimes \mathcal{H}_{k}^{R}, \mathcal{K}_{k}^{L} \otimes \mathcal{K}_{k}^{R}\right)$ and it can be factorized into the form $\Phi_{k}=\operatorname{Ad}_{U_{k}} \otimes \Phi_{k}^{R}$, where $U_{k}: \mathcal{H}_{k}^{L} \longrightarrow \mathcal{K}_{k}^{L}$ is unitary operator and $\Phi_{k}^{R} \in \mathrm{T}\left(\mathcal{H}_{k}^{R}, \mathcal{K}_{k}^{R}\right)$ is stochastic, $k=1, \ldots, K$.

(3) The state $\rho$ decomposes as $\rho=\bigoplus_{k=1}^{K} p_{k} \rho_{k}^{L} \otimes \omega_{k}^{R}, \sigma=\bigoplus_{k=1}^{K} q_{k} \sigma_{k}^{L} \otimes \omega_{k}^{R}$, where all the operators are density operators, and $\left\{p_{k}\right\}_{k=1}^{K}$ and $\left\{q_{k}\right\}_{k=1}^{K}$ are probability distributions.

Therefore, it follows that the result can be proved by the above decomposition of $\Phi$.

Remark 2.4. In [13], it was shown that $S(\Phi(\rho))=S(\rho)$ if and only if $\Phi^{\dagger} \circ \Phi(\rho)=\rho$ while the explicit construction of the state $\rho$ and the quantum operation $\Phi$ are given. We can employ the mentioned result to give an explicit construction for $T, \mathbf{p}$ in the identity: $H(T \mathbf{p})=\mathrm{H}(\mathbf{p})$. The proof is trivial and is omitted.

\section{Relative entropy of stochastic matrices}

In this section, the entropy of stochastic matrices is discussed. For the entropy of stochastic matrices, more details can be found in [12]. We will go deeper within the entropy concerning stochastic matrices and derive some conditions on the (strong) additivity for the stochastic matrices. The notion of relative entropy of stochastic matrices is introduced by mimicking quantum relative entropy. Some properties of this concept are listed and the connection between the entropy of the stochastic quantum operations and that of stochastic matrices are discussed.

To be specific, for any $N \times N$ stochastic matrix $T=\left[t_{\mu v}\right]$, the weighted entropy [12] of $T$ by a probability vector $\mathbf{p}=\left[p_{1}, \ldots, p_{N}\right]^{\top}$ is defined by $\mathrm{H}_{\mathbf{p}}(T)=\sum_{v=1}^{N} p_{v} \mathrm{H}\left(\mathbf{t}_{v}\right)$, where $T=\left[\mathbf{t}_{1}, \ldots, \mathbf{t}_{N}\right]$ 
and $\mathbf{t}_{v}=\left[t_{1 v}, \ldots, t_{N v}\right]^{\top}$ is the $v$ th column vector of $T$. In particular, $\mathrm{H}(T)=\frac{1}{N} \sum_{v=1}^{N} \mathrm{H}\left(\mathbf{t}_{v}\right)$ is defined for $\mathbf{p}=\frac{1}{N}[1, \ldots, 1]^{\top}$.

For any two $N \times N$ stochastic matrices $A$ and $B$, the relative entropy between $A$ and $B$ with respect to a probability vector $\mathbf{p}=\left[p_{1}, \ldots, p_{N}\right]^{\top}$ is defined by $\mathrm{H}_{\mathbf{p}}(A \| B)=\sum_{v=1}^{N} p_{v} \mathrm{H}\left(\mathbf{a}_{v} \| \mathbf{b}_{v}\right)$, where $\mathbf{a}_{v}$ and $\mathbf{b}_{v}$ are the $v$ th column vectors of $A$ and $B$, respectively. Similarly, $\mathrm{H}(A \| B)=$ $\frac{1}{N} \sum_{v=1}^{N} \mathrm{H}\left(\mathbf{a}_{v} \| \mathbf{b}_{v}\right)$ is defined for $\mathbf{p}=\frac{1}{N}[1, \ldots, 1]^{\top}$.

The following conclusions are immediate. That is, $\mathrm{H}_{\mathbf{p}}(\cdot)$ is a nonnegative and concave function; $\mathrm{H}_{\mathbf{p}}(\cdot \| \cdot)$ is a jointly convex function.

In what follows, the monotonicity of relative entropy of stochastic matrices is obtained.

Theorem 3.1. If $T, A, B$ are all $N \times N$ stochastic matrices, then

$$
\mathrm{H}_{\mathbf{p}}(T A \| T B) \leqslant \mathrm{H}_{\mathbf{p}}(A \| B),
$$

where $\mathbf{p}$ is an $\mathrm{N}$-dimensional probability vector. Moreover, if all the components of $\mathbf{p}$ are positive, then

$$
\mathrm{H}_{\mathbf{p}}(T A \| T B)=\mathrm{H}_{\mathbf{p}}(A \| B)
$$

if and only if the following conditions hold:

(i) $\mathbf{a}_{j}=\bigoplus_{k=1}^{K} \mu_{k}^{(j)} \mathbf{p}_{k}^{(j)} \otimes \mathbf{r}_{k}$ and $\mathbf{b}_{j}=\bigoplus_{k=1}^{K} v_{k}^{(j)} \mathbf{q}_{k}^{(j)} \otimes \mathbf{r}_{k}$, where $\mathbf{p}_{k}^{(j)}, \mathbf{q}_{k}^{(j)}$ denote $m_{k}$-dimensional probability vectors, and $\mathbf{r}_{k}$ are $n_{k}$-dimensional probability vectors and $\forall k: \mu_{k}, v_{k} \geqslant 0, \sum_{k=1}^{K} \mu_{k}^{(j)}=$ $\sum_{k=1}^{K} v_{k}^{(j)}=1$ for each $j=1, \ldots, N, \sum_{k=1}^{K} m_{k} n_{k}=N$;

(ii) $T=\bigoplus_{k=1}^{K} \pi_{k} \otimes T_{k}$, where $\pi_{k} \in \mathbf{S}_{m_{k}}$ and $T_{k}$ is $n_{k} \times n_{k}$ stochastic matrix for each $k$.

Proof. By the definition of relative entropy for stochastic matrices, it follows that

$$
\mathrm{H}_{p}(A \| B)=\sum_{j=1}^{N} p_{j} \mathrm{H}\left(\mathbf{a}_{j}|| \mathbf{b}_{j}\right),
$$

where $\mathbf{a}_{j}=\left[a_{1 j}, \ldots, a_{N j}\right]^{\top}$ and $\mathbf{b}_{j}=\left[b_{1 j}, \ldots, b_{N j}\right]^{\top}$ are $j$ th columns of $A$ and $B$, respectively. Now

$$
\begin{aligned}
\mathrm{H}_{\mathbf{p}}(T A \| T B) & =\sum_{j=1}^{N} p_{j} \mathrm{H}\left((T A)_{j} \|(T B)_{j}\right)=\sum_{j=1}^{N} p_{j} \mathrm{H}\left(T \mathbf{a}_{j} \| T \mathbf{b}_{j}\right) \\
& \leqslant \sum_{j=1}^{N} p_{j} \mathrm{H}\left(\mathbf{a}_{j} \| \mathbf{b}_{j}\right)=\mathrm{H}_{\mathbf{p}}(A \| B)
\end{aligned}
$$

Thus it follows from the above process in the proof that when the components of $p$ are all positive, $\mathrm{H}_{\mathbf{p}}(T A \| T B)=\mathrm{H}_{\mathbf{p}}(A \| B)$ if and only if $\mathrm{H}\left(T \mathbf{a}_{j} \| T \mathbf{b}_{j}\right)=\mathrm{H}\left(\mathbf{a}_{j} \| \mathbf{b}_{j}\right)$ for each $j$. By Theorem 2.3, the equality condition can be concluded immediately. 
Remark 3.2. Now denote $L^{(k)}=\left[\mathbf{p}_{k}^{(1)}, \ldots, \mathbf{p}_{k}^{(N)}\right]$ and $R^{(k)}=\left[\mathbf{q}_{k}^{(1)}, \ldots, \mathbf{q}_{k}^{(N)}\right]$. Let $E^{(k)}=\operatorname{Diag}\left[\mu_{k}^{(1)}, \ldots, \mu_{k}^{(N)}\right]$ and $F^{(k)}=\operatorname{Diag}\left[v_{k}^{(1)}, \ldots, v_{k}^{(N)}\right]$. The explicit forms of $A, B$ can be written as

$$
A=\bigoplus_{k=1}^{K} E^{(k)} L^{(k)} \otimes \mathbf{r}_{k} \text { and } B=\bigoplus_{k=1}^{K} F^{(k)} R^{(k)} \otimes \mathbf{r}_{k},
$$

where $L^{(k)}$ and $R^{(k)}$ are any stochastic matrices, where $k=1, \ldots, K$. Furthermore, $\sum_{k=1}^{K} E^{(k)}=$ $\sum_{k=1}^{K} F^{(k)}=\mathbb{1}$.

For a finite collection $\left\{B^{(i)}\right\}$ of $N \times N$ stochastic matrices, denote $\bar{B}=\sum_{i} \lambda_{i} B^{(i)}$, where $\left\{\lambda_{i}\right\}$ is a probability vector. The $\chi$-quantity for $\left\{B^{(i)}\right\}$ is defined by $\chi_{\mathbf{p}}\left(\left\{\lambda_{i}, B^{(i)}\right\}\right)=\sum_{i} \lambda_{i} \mathrm{H}_{\mathbf{p}}\left(B^{(i)} \| \bar{B}\right)$, where $\mathbf{p}$ is a probability vector. It is easily seen from Theorem 3.1 that

(i) $\chi_{\mathbf{p}}\left(\left\{\lambda_{i}, B^{(i)}\right\}\right)=\mathrm{H}_{\mathbf{p}}\left(\sum_{i} \lambda_{i} B^{(i)}\right)-\sum_{i} \lambda_{i} \mathrm{H}_{\mathbf{p}}\left(B^{(i)}\right)$;

(ii) $\sum_{i} \lambda_{i} \mathrm{H}_{\mathbf{p}}\left(B^{(i)} \| D\right)=\chi_{\mathbf{p}}\left(\left\{\lambda_{i}, B^{(i)}\right\}\right)+\mathrm{H}_{\mathbf{p}}(\bar{B} \| D)$, i.e., $\sum_{i} \lambda_{i} \mathrm{H}_{\mathbf{p}}\left(B^{(i)} \| D\right)=\sum_{i} \lambda_{i} \mathrm{H}_{\mathbf{p}}\left(B^{(i)} \| \bar{B}\right)+$ $\mathrm{H}_{\mathbf{p}}(\bar{B} \| D)$, where $D$ is an $N \times N$ stochastic matrix and $p$ is an $N$-dimensional probability vector.

(iii) Assume that $T$ is an $N \times N$ stochastic matrix. Then $\chi_{\mathbf{p}}\left(\left\{\lambda_{i}, T B^{(i)}\right\}\right) \leqslant \chi_{\mathbf{p}}\left(\left\{\lambda_{i}, B^{(i)}\right\}\right)$ if and only if $\mathrm{H}_{\mathbf{p}}(T B)-\mathrm{H}_{\mathbf{p}}(B)$ is a convex function in its argument stochastic matrix $B$; moreover, $\chi_{\mathbf{p}}\left(\left\{\lambda_{i}, T B^{(i)}\right\}\right) \leqslant \chi_{\mathbf{p}}\left(\left\{\lambda_{i}, B^{(i)}\right\}\right)$.

In [12], W. Słomczyński obtained that given any $N \times N$ stochastic matrices $X, Y, Z$ for which $\mathbf{p}$ is their common invariant probability vector, i.e. $X \mathbf{p}=Y \mathbf{p}=Z \mathbf{p}=\mathbf{p}$. Then :

(i) $\mathrm{H}_{\mathbf{p}}(Y) \leqslant \mathrm{H}_{\mathbf{p}}(X Y) \leqslant \mathrm{H}_{\mathbf{p}}(X)+\mathrm{H}_{\mathbf{p}}(Y)$;

(ii) $\mathrm{H}_{\mathbf{p}}(X Y Z)+\mathrm{H}_{\mathbf{p}}(Y) \leqslant \mathrm{H}_{\mathbf{p}}(X Y)+\mathrm{H}_{\mathbf{p}}(Y Z)$.

The following result is to deal with the saturation of the above two inequalities.

Proposition 3.3. (i) If $T \in \mathbf{B}_{N}, A$ is an $N \times N$ stochastic matrix and $\mathbf{p}$ is an $N$-dimensional probability vector with all positive components, then $\mathrm{H}_{\mathbf{p}}(T A)=\mathrm{H}_{\mathbf{p}}(A)$ if and only if $T^{\top} T A=A$;

(ii) If $X=X_{L} \otimes \pi_{R}$ and $Y=\pi_{L} \otimes Y_{R}$ for $X_{L}$ being stochastic matrix of size $m \times m, \pi_{L} \in \mathbf{S}_{m}, Y_{R}$ being stochastic matrix of size $n \times n, \pi_{R} \in \mathbf{S}_{n}$, then $\mathrm{H}(X Y)=\mathrm{H}(X)+\mathrm{H}(Y)$;

(iii) If $X=\bigoplus_{k=1}^{K} X_{k}^{L} \otimes \pi_{k}^{R}, Y=\bigoplus_{k=1}^{K} Y_{k}^{L} \otimes Y_{k}^{R}$ and $Z=\bigoplus_{k=1}^{K} \pi_{k}^{L} \otimes Z_{k}^{R}$ for $X_{k}^{L}$ being stochastic matrix of size $m_{k} \times m_{k}, \pi_{k}^{L} \in \mathbf{S}_{m_{k}}, Y_{k}^{R}$ being stochastic matrix of size $n_{k} \times n_{k}, \pi_{k}^{R} \in \mathbf{S}_{n_{k}}$, then $\mathrm{H}(X Y Z)+\mathrm{H}(Y)=\mathrm{H}(X Y)+\mathrm{H}(Y Z)$.

Proof. (i) Since each component $p_{j}$ is positive, it follows that $\mathrm{H}_{\mathbf{p}}(T A)=\mathrm{H}_{\mathbf{p}}(A)$ if and only if $\mathrm{H}\left(T \mathbf{a}_{j}\right)=\mathrm{H}\left(\mathbf{a}_{j}\right)$ for every $j$, where all $\mathbf{a}_{j}$ 's are the $j$ th column vector of $A$. By the result in [9, 13], i.e. for $B \in \mathbf{B}_{N}, \mathrm{H}(B \mathbf{p})=\mathrm{H}(\mathbf{p})$ if and only if $B^{\top} B \mathbf{p}=\mathbf{p}$, we get that $\mathrm{H}\left(T \mathbf{a}_{j}\right)=\mathrm{H}\left(\mathbf{a}_{j}\right)$ for every $j$ if and only if $T^{\top} T \mathbf{a}_{j}=\mathbf{a}_{j}$ for all $j$; that is, the proof is concluded. 
(ii) Since $X Y=X_{L} \pi_{L} \otimes \pi_{R} Y_{R}$, it follows that $\mathrm{H}(X Y)=\mathrm{H}\left(X_{L} \pi_{L} \otimes \pi_{R} Y_{R}\right)=\mathrm{H}\left(X_{L}\right)+\mathrm{H}\left(Y_{R}\right)$, which implies the conclusion.

(iii) Since

$$
X Y Z=\bigoplus_{k=1}^{K} X_{k}^{L} Y_{k}^{L} \pi_{k}^{L} \otimes \pi_{k}^{R} Y_{k}^{R} Z_{k}^{R}
$$

it follows that

$$
\begin{aligned}
\mathrm{H}(X Y Z) & =\sum_{k} \lambda_{k}\left[\mathrm{H}\left(X_{k}^{L} Y_{k}^{L}\right)+\mathrm{H}\left(Y_{k}^{R} Z_{k}^{R}\right)\right] \\
\mathrm{H}(X Y) & =\sum_{k} \lambda_{k}\left[\mathrm{H}\left(X_{k}^{L} Y_{k}^{L}\right)+\mathrm{H}\left(Y_{k}^{R}\right)\right], \\
\mathrm{H}(Y Z) & =\sum_{k} \lambda_{k}\left[\mathrm{H}\left(Y_{k}^{L}\right)+\mathrm{H}\left(Y_{k}^{R} Z_{k}^{R}\right)\right], \\
\mathrm{H}(Y) & =\sum_{k} \lambda_{k}\left[\mathrm{H}\left(Y_{k}^{L}\right)+\mathrm{H}\left(Y_{k}^{R}\right)\right],
\end{aligned}
$$

where $\lambda_{k}=m_{k} n_{k} / N$ and $\sum_{k} m_{k} n_{k}=N$. Combining all these expressions gives the desired result.

\section{The relationship between quantum operations and bi-stochastic ma- trices}

For any CP super-operators $\Phi$ and $\Psi$, with corresponding Kraus representations: $\Phi=\sum_{i} \operatorname{Ad}_{M_{i}}$ and $\Psi=\sum_{j} \mathbf{A d}_{N_{j}}$, respectively, it is easily seen that $\Phi \otimes \Psi=\sum_{i, j} \operatorname{Ad}_{M_{i} \otimes N_{j}}$, and $J(\Phi \otimes \Psi)=$ $J(\Phi) \otimes J(\Psi)$; denote $\Psi^{\top}=\sum_{j} \mathbf{A d}_{N_{j}^{\top}}$. Then

$$
J(\Phi \circ \Psi)=\Phi \otimes \mathbb{1}(J(\Psi))=\mathbb{1} \otimes \Psi^{\top}(J(\Phi))=\Phi \otimes \Psi^{\top}\left(\operatorname{vec}(\mathbb{1}) \operatorname{vec}(\mathbb{1})^{\dagger}\right) .
$$

Let $\Phi, \Psi \in \mathrm{CP}(\mathcal{H})$ be stochastic. The relative entropy between $\Phi$ and $\Psi$ is defined by

$$
S(\Phi \| \Psi) \stackrel{\text { def }}{=} S(\rho(\Phi) \| \rho(\Psi))
$$

If $\Lambda \in \mathrm{CP}(\mathcal{H})$ is also bi-stochastic, then

$$
\mathrm{S}(\Lambda \circ \Phi \| \Lambda \circ \Psi) \leqslant \mathrm{S}(\Phi \| \Psi)
$$

This can be seen easily from the Lemma 2.1. Indeed,

$$
\mathrm{S}\left(\Lambda \otimes \mathbb{1}_{\mathrm{L}(\mathcal{H})}(\rho(\Phi)) \| \Lambda \otimes \mathbb{1}_{\mathrm{L}(\mathcal{H})}(\rho(\Psi))\right) \leqslant \mathrm{S}(\rho(\Phi) \| \rho(\Psi))=\mathrm{S}(\Phi \| \Psi)
$$


since $\Lambda \otimes \mathbb{1}_{\mathrm{L}(\mathcal{H})}$ is bi-stochastic whenever $\Lambda$ is bi-stochastic.

Assume that $\Phi$ is a CP stochastic super-operator for which the Kraus decomposition can be written as $\Phi=\sum_{\mu} \mathbf{A d}_{T_{\mu}}$. Define the Kraus matrix [1] for $\Phi$ as

$$
B(\Phi):=\sum_{\mu} T_{\mu} \bullet T_{\mu}^{*}
$$

where $\bullet$ denotes Shur product of matrices and $*$ means that entry-wise complex conjugate of a matrix. Hence the $(i, j)$ th entry $b_{i j}$ of $B$ can be described by $b_{i j}=\sum_{\mu} t_{i j}^{\mu} t_{i j}^{\mu}$, where $T_{\mu}=\left[t_{i j}^{\mu}\right]$ and the bar means the complex conjugate of complex numbers.

For any two Hermitian matrices $X$ and $Y, X$ is majorized by $Y$, denoted by $X \prec Y$, if there is a CP bi-stochastic super-operator $\Phi$ such that $X=\Phi(Y)$. The well-known Shur's theorem states that $\operatorname{Diag}(X) \prec X$ for any square matrix $X$, see [1]. Thus for any bi-stochastic quantum operation $\Lambda$, it follows that $\Lambda(\rho) \prec \rho$. Moreover,

$$
J(\Phi \circ \Psi) \prec J(\Phi), \quad J(\Phi \circ \Psi) \prec J(\Psi)
$$

for any two bi-stochastic quantum operations $\Phi$ and $\Psi$.

In what follows, some properties of Kraus matrices are listed below.

Proposition 4.1. (i) For a given (bi-)stochastic super-operator $\Phi \in \mathrm{CP}(\mathcal{H}), B(\Phi)$ is a (bi-)stochastic matrix.

(ii) $B(\Phi)$ is well-defined, i.e., it is independent of the different Kraus decompositions for $\Phi$ and just depends on $\Phi$ itself.

(iii) $B(\Phi)$ is a convex function with respect to argument $\Phi$, i.e., $B\left(t \Phi_{1}+(1-t) \Phi_{2}\right)=t B\left(\Phi_{1}\right)+(1-t) B\left(\Phi_{2}\right)$ for any $\Phi_{1}, \Phi_{2}$ and all $t \in[0,1]$.

(iv) Denote $\mathscr{M}(B)=\{\Phi \mid \Phi \in \mathrm{T}(\mathcal{H})$ is $C P$ stochastic super-operator and $B(\Phi)=B\}$. Then $\mathscr{M}(B)$ is a nonempty convex set.

(v) $B\left(\Theta_{1} \otimes \Theta_{2}\right)=B\left(\Theta_{1}\right) \otimes B\left(\Theta_{2}\right)$ for any stochastic quantum operations $\Theta_{1}$ and $\Theta_{2}$.

(vi) Assume that $\mathcal{H}$ and $\mathcal{K}$ are $M$ and $N$ dimensional Hilbert spaces, respectively. If $\Lambda \in \mathrm{T}(\mathcal{H} \otimes \mathcal{K})$ is $C P$ and stochastic and can be described by $\Lambda=\sum_{k} \lambda_{k} \Phi_{k} \otimes \Psi_{k}$, where $\left\{\Phi_{k}\right\} \in \mathrm{T}(\mathcal{H})$ and $\left\{\Psi_{k}\right\} \in$ $\mathrm{T}(\mathcal{K})$ are two collections of $C P$ stochastic super-operators, then $B(\Lambda)=\sum_{k} \lambda_{k} B\left(\Phi_{k}\right) \otimes B\left(\Psi_{k}\right)$, where $\lambda=\left\{\lambda_{k}\right\}_{k}$ is a finite probability vector.

Proof. (i) The proof is trivial. 
(ii) Assume that $\Phi=\sum_{\mu=1}^{N^{2}} \mathbf{A d}_{E_{\mu}}=\sum_{v=1}^{N^{2}} \mathbf{A d}_{F_{v}}$. By the unitary freedom of quantum operations, there is a $N^{2} \times N^{2}$ unitary matrix $U=\left[u_{\mu \nu}\right]$ such that $E_{\mu}=\sum_{v=1}^{N^{2}} u_{\mu \nu} F_{v}$. Then

$$
\begin{aligned}
\sum_{\mu=1}^{N^{2}} E_{\mu} \bullet E_{\mu}^{*} & =\sum_{\mu=1}^{N^{2}}\left(\sum_{v=1}^{N^{2}} u_{\mu \nu} F_{v}\right) \bullet\left(\sum_{\kappa=1}^{N^{2}} u_{\mu \kappa} F_{\kappa}\right)^{*}=\sum_{\nu, \kappa=1}^{N^{2}}\left(\sum_{\mu=1}^{N^{2}} u_{\mu \nu} \bar{u}_{\mu \kappa}\right) F_{v} \bullet F_{\kappa}^{*} \\
& =\sum_{v, \kappa=1}^{N^{2}} \delta_{v \kappa} F_{v} \bullet F_{\kappa}^{*}=\sum_{v=1}^{N^{2}} F_{v} \bullet F_{v}^{*}
\end{aligned}
$$

which implies that $B(\Phi)$ is well-defined.

(iii) Choose any two stochastic quantum operations $\Phi_{1}$ and $\Phi_{2}$ with their corresponding Kraus decomposition: $\Phi_{1}=\sum_{\mu} \mathbf{A d}_{S_{\mu}}$ and $\Phi_{2}=\sum_{v} \mathbf{A d}_{T_{v}}$. Let $t \in[0,1]$. Then the Kraus decomposition for $t \Phi_{1}+(1-t) \Phi_{2}$ is $t \Phi_{1}+(1-t) \Phi_{2}=t \sum_{\mu} \mathbf{A d}_{S_{\mu}}+(1-t) \sum_{v} \mathbf{A d}_{T_{v}}$, which implies that the Kraus matrix for $t \Phi_{1}+(1-t) \Phi_{2}$ is

$$
\begin{aligned}
B\left(t \Phi_{1}+(1-t) \Phi_{2}\right) & =\sum_{\mu}\left(\sqrt{t} S_{\mu}\right) \bullet\left(\sqrt{t} S_{\mu}^{*}\right)+\sum_{v}\left(\sqrt{1-t} T_{v}\right) \bullet\left(\sqrt{1-t} T_{v}^{*}\right) \\
& =t \sum_{\mu} S_{\mu} \bullet S_{\mu}^{*}+(1-t) \sum_{v} T_{v} \bullet T_{v}^{*} \\
& =t B\left(\Phi_{1}\right)+(1-t) B\left(\Phi_{2}\right) .
\end{aligned}
$$

(iv) If $\Psi_{1}, \Psi_{2} \in \mathscr{M}(B)$, it follows from the result of (iii) that $B\left(t \Psi_{1}+(1-t) \Psi_{2}\right)=t B\left(\Psi_{1}\right)+(1-$ t) $B\left(\Psi_{2}\right)=B$ since $B\left(\Psi_{1}\right)=B\left(\Psi_{2}\right)=B$, which implies $t \Psi_{1}+(1-t) \Psi_{2} \in \mathscr{M}(B)$. The fact that $\mathscr{M}(B)$ is not empty is clear.

(v) Let the Kraus decompositions for $\Theta_{1}$ and $\Theta_{2}$ are $\Theta_{1}=\sum_{m} \mathbf{A d}_{S_{m}}$ and $\Theta_{2}=\sum_{\mu} \mathbf{A d}_{T_{\mu}}$. Then $\Theta_{1} \otimes \Theta_{2}=\sum_{m, \mu} A d_{S_{m} \otimes T_{\mu}}$. Now

$$
\begin{aligned}
B\left(\Theta_{1} \otimes \Theta_{2}\right) & =\sum_{m, \mu}\left(S_{m} \otimes T_{\mu}\right) \bullet\left(S_{m}^{*} \otimes T_{\mu}^{*}\right)=\left(\sum_{m} S_{m} \bullet S_{m}^{*}\right) \otimes\left(\sum_{\mu} T_{\mu} \bullet T_{\mu}^{*}\right) \\
& =B\left(\Theta_{1}\right) \otimes B\left(\Theta_{2}\right) .
\end{aligned}
$$

(vi) It follows trivially from combining the above conclusions (iii) and (v).

Remark 4.2. In the above Proposition 4.1(iv), it is known that $\mathscr{M}(B)$ is a nonempty convex set. In fact, it is also compact. Thus the question naturally arises: what are the extreme points of $\mathscr{M}(B)$ ? Note that in [8], Parthasarathy gave a characterization of extremal quantum states of composite systems with fixed marginal states. Subsequently, Rudolph gave an another characterization about it in [11]. Therefore, our question can be described in terms of the language as in [8, 11] under the additional condition that the diagonals of the Jamiołkowski state are fixed. 
Remark 4.3. Generally speaking, $B(\Phi \circ \Psi) \neq B(\Phi) B(\Psi)$ for two stochastic super-operators $\Phi, \Psi \in \mathrm{CP}(\mathcal{H})$. But there is a class of specific examples in which the inequality sign can be replaced by an equal sign. Indeed, assume that $\mathcal{H}$ is an $N$-dimensional Hilbert space and $\Phi, \Psi \in \mathrm{CP}(\mathcal{H})$ for which

$$
J(\Phi)=\sum_{m, \mu=1}^{N} p_{m \mu}|m \mu\rangle\langle m \mu| \text { and } J(\Psi)=\sum_{m \mu=1}^{N} q_{m, \mu}|m \mu\rangle\langle m \mu| .
$$

By the stochasticity, $\sum_{\mu=1}^{N} p_{m \mu}=1$ and $\sum_{m=1}^{N} p_{m \mu}=1 ; \sum_{\mu=1}^{N} q_{m \mu}=1$ and $\sum_{m=1}^{N} q_{m \mu}=1$. Then $J(\Phi \circ \Psi)=\sum_{m, \mu=1}^{N}[B(\Phi) B(\Psi)]_{m \mu}|m \mu\rangle\langle m \mu|$, where $B(\Phi)=\left[p_{m \mu}\right]$ and $B(\Psi)=\left[q_{m \mu}\right]$, which implies that

$$
B(\Phi \circ \Psi)=B(\Phi) B(\Psi), \quad B(\Psi \circ \Phi)=B(\Psi) B(\Phi)
$$

and

$$
\mathrm{S}(\Phi \circ \Psi)=\mathrm{H}(B(\Phi \circ \Psi))+\log N, \quad \mathrm{~S}(\Psi \circ \Phi)=\mathrm{H}(B(\Psi \circ \Phi))+\log N
$$

Now

$$
\mathrm{S}^{\operatorname{map}}(\Phi)+\mathrm{S}^{\operatorname{map}}(\Psi)-\mathrm{S}^{\operatorname{map}}(\Phi \circ \Psi)=\mathrm{H}(B(\Phi))+\mathrm{H}(B(\Psi))-\mathrm{H}(B(\Psi) B(\Phi))+\log N
$$

This fact shows that if both $J(\Phi)$ and $J(\Psi)$ are diagonal, then $B(\Phi \circ \Psi)=B(\Phi) B(\Psi)$. There is a question which can be formulated as follows: what is a sufficient and necessary condition for $B(\Phi \circ \Psi)=B(\Phi) B(\Psi)$ for stochastic super-operators $\Phi, \Psi \in \mathrm{CP}(\mathcal{H})$. It is also conjectured that $\mathrm{H}_{\mathbf{p}}(B(\Phi \circ \Psi)) \leqslant \mathrm{H}_{\mathbf{p}}(B(\Phi) B(\Psi))$ for any stochastic super-operators $\Phi, \Psi \in \mathrm{CP}(\mathcal{H})$, where $\mathbf{p}$ is any $N$-dimensional probability vector.

Proposition 4.4. Assume that $\mathcal{H}$ is a $\mathrm{N}$-dimensional Hilbert space.

(i) If $\Phi \in \mathrm{CP}(\mathcal{H})$ is stochastic, then: $\mathrm{S}^{\operatorname{map}}(\Phi) \leqslant \mathrm{H}(B(\Phi))+\log N$;

(ii) If $\Phi, \Psi \in \mathrm{CP}(\mathcal{H})$ is stochastic, then: $\mathrm{H}(B(\Phi) \| B(\Psi)) \leqslant \mathrm{S}(\Phi \| \Psi)$;

Proof. (i) By Shur's lemma, it follows that $\operatorname{Diag}(J(\Phi)) \prec J(\Phi)$ which is equivalent to $\operatorname{Diag}(\rho(\Phi)) \prec$ $\rho(\Phi)$. Since $\langle m|B(\Phi)| \mu\rangle=\langle m \mu|J(\Phi)| m \mu\rangle$, it can be seen that

$$
\mathrm{S}^{\mathrm{map}}(\Phi)=\mathrm{S}(\rho(\Phi)) \leqslant \mathrm{S}(\operatorname{Diag}(\rho(\Phi)))=\mathrm{H}(B(\Phi))+\log N .
$$

Furthermore, $\mathrm{S}^{\operatorname{map}}(\Phi)=\mathrm{H}(B(\Phi))+\log N$ when $\Phi$ is represented by a diagonal dynamical matrix $J(\Phi)$. 
(ii) There exists a CP bi-stochastic super-operator $\Lambda$ such that $\Lambda(\rho)=\operatorname{Diag}(\rho)$ since $\operatorname{Diag}(\rho) \prec$ $\rho$ which follows from Shur's lemma. Thus it follows from Lemma 2.1 that

$$
\begin{aligned}
\mathrm{S}(\Phi \| \Psi) & =\mathrm{S}(\rho(\Phi) \| \rho(\Psi)) \geqslant \mathrm{S}(\operatorname{Diag}[\rho(\Phi)] \| \operatorname{Diag}[\rho(\Psi)]) \\
& =\frac{1}{N} \sum_{j=1}^{N} \mathrm{H}\left(B(\Phi)_{j} \| B(\Psi)_{j}\right)=\mathrm{H}(B(\Phi) \| B(\Psi))
\end{aligned}
$$

Remark 4.5. Recently, Roga et al. studied entropic uncertainty relations for quantum operations in [14] and related bounds on the map entropy were also obtained.

Let $\widetilde{s}(\Phi)=\mathrm{H}(B(\Phi))-\mathrm{S}^{\mathrm{map}}(\Phi)$ for stochastic super-operator $\Phi \in \mathrm{CP}(\mathcal{H})$. Then for a collection $\left\{\Phi_{k}\right\}$ of stochastic super-operator in $\mathrm{CP}(\mathcal{H})$ such that $\Phi=\sum_{k} \lambda_{k} \Phi_{k}, \chi\left(\left\{\lambda_{k}, B\left(\Phi_{k}\right)\right\}\right) \leqslant$ $\chi\left(\left\{\lambda_{k}, \Phi_{k}\right\}\right)$ if and only if $\widetilde{s}\left(\sum_{k} \lambda_{k} \Phi_{k}\right) \leqslant \sum_{k} \lambda_{k} \widetilde{s}\left(\Phi_{k}\right)$; i.e., $\widetilde{s}(\Phi)$ is a convex function in its argument $\Phi$.

\section{Acknowledgement}

The author would like to thank the anonymous referee(s) for valuable comments and to thank KMR Audenaert for pointing out some misprints appearing in this paper. LZ is also grateful for funding from Hangzhou Dianzi University (Grant No. KYS075612038).

\section{References}

[1] I. Bengtsson and K. Życzkowski, Geometry of quantum states, Cambridge University Press, pp. 315 (2006).

[2] M. D. Choi, Completely positive linear maps on complex matrices, Linear algebra and its applications, 10: 285-290 (1975).

[3] K. Kraus, States, effects, and operations : fundamental notions of quantum theory, Publisher: Springer-Verlag (1983).

[4] C. K. Li and Y. T. Poon, Interpolation problems by completely positive maps, Linear and Multilinear Algebra 59: 1159-1170 (2011).

[5] G. Lindblad, Completely Positive Maps and Entropy Inequalities, Commun. math. Phys. 40: 147-151 (1975). 
[6] M. Mosonyi and D. Petz: Structure of Sufficient Quantum Coarse Grainings, Lett. Math. Phys. 68: 19-30 (2004).

[7] M. A. Nielsen and I. L. Chuang, Quuntum Computation and Quantum Information, Cambridge University Press, Cambridge (2000).

[8] K.R. Parthasarathy, Extremal quantum states in coupled systems, Ann. I. H. Poincaré-PR 41: 257-268 (2005).

[9] A. B. Poritz and J. A. Poritz: On entropy-preserving stochastic averages, Linear Algebra and its Applications 434: 1425-1442 (2011).

[10] W. Roga, M. Fannes and K. Życzkowski, Composition of quantum states and dynamical subadditivity. J. Phys. A: Math. Theor. 41, 035305 (2008).

[11] O. Rudolph, On extremal quantum states of composite systems with fixed marginas, J. Math. Phys. 45: 4035-4041 (2004).

[12] W. Słomczyński, Subadditivity of entropy for stochastic matrices, Open\& Information Dyn. 9: 201-206 (2002).

[13] L. Zhang and J. Wu, Von Neumann Entropy-Preserving Quantum Operations, Phys. Lett. A 375(47): 4163-4165 (2011).

[14] W. Roga, Z. Puchała, L. Rudnicki, K. Życzkowski, Entropic uncertainty relation for quantum operations, arXiv:1206.2536 